\title{
Ophthalmologische Gesellschaft in Wien
}

Sitzungsbericht vom 7. Juli 1924

Demonstrati onen. 1. Weizenblaü: Stelliuigsanomalie der Oberlider infolge Tarsusverdüunuug. Bei einer 18jähr. Patientin besteht ein beiderseitiges Ektropium der Oberlider. Die Lidränder sind ganz nach. oben gekehrt, und man sieht schon von vorne einen schmalen Saum der Lidinnenfläche. Die Stellung der Unter-lider ist bis auf eine geringe Eversion der Lidränder normal. Die Bindehaut der Unterlider ist ganz normal. Im Tarsus selbst sieht man zwei weißglänzende, ca. $4-6 \mathrm{~mm}$ lange und $0,5 \mathrm{~mm}$ bis $1 \mathrm{~mm}$ breite, zueinander parallele Streiíen, die im Sulcus subtarsalis liegen. In ihrem Bereiche ist eine Unterbrechung der Zeichnung (Meibomsche Drüsen) sichtbar. An den Oberlidern sind ähnliche, aber 338 Ophthalmologische Gesellschaft in Wien.

kürzere Streifen, auch im Sulcus subtarsaîis, vorhanden, hier kommen noch gelblich-opake, schräge und vertikale Bindehautnarben hinzu, die die weißen Streifen des Tarsus überkreuzen. Die übrige Bindehaut ist ganz normal.

Weiter besteht eine beiderseitige, abgelauiene Keratitis parenchymatosa, eine Anisokorie, Entrundung der Pupillen und träge Lichtreaktion, eine Cha-rioiditis anterior.

Vis R -10s o 3,5 cyl. lõ0712-6/9?? LA -11s 6/9.

Wenn man versucht, die Oberlider umzustülpen, so gelingt dies kaum, denn der Tarsus erweist sich als so dünn, daß er sich unter den Fingern rollt. Bei dieser Tarsusbeschaffenheit ist es möglich, daß die Sehnenfasern des Muse, levator, die zur Haut des Oberlides ziehen, dieses nach auswärts drehen, wozu als begünstigendes Moment der prominente, myope Bulbus hinzukommt. Die Veränderungen des Tarsus lassen, bei den anderen Zeichen einer Lues congenita der Augen, an eine abgelauiene Tarsitis luetica denken, da sowohl anamnestisch als auch objektiv jeder andere narbenbildende Prozeß an Bindehaut und Tarsus ausgeschlossen werden kann. Dazu kommt noch die Eigen-art der Narben größtenteils im Tarsus bei normaler Bindehaut zu liegen. Mutter der Pat. hatte drei Abortus, zwei Partus, der Bruder der Pat. ist angeblich gesund. Die Pat. ist sehr zart, war als Kind immer kränklich. Die Zähne sind verkümmert, stehen in großen Abständen. Wassermann-Reaktion konnte aus äußeren Gründen nicht angestellt werden.

2. Bachstez: Auîtreten einer Ptosis nach Zal $\pi$ iextraktiou.

22jährige Patientin, bei der wenige Tage nach einer ganz glatten Zahn-extraktion vor 2 Jahren eine Ptosis auf der Seite der Extraktion aufgetreten war. Patientin steht seit mehr als einem Jahr an der Klinik in Beobachtung, die Ptosis ist eine echte schlaffe und vollkommen stationär. Das zeitliche Auîtreten nach der Extraktion 3/8ist sichergestellt, alle anderen ätiologischen Untersuchungen, die jetzt neuerlich wiederholt wurden, sind negativ. Nach der Extraktion kein Fieber, keine AUgemeinerscheinungen. Die Wundheilung ohne Verzögerung. Leichte Extraktion, kein gleichzeitiges Trauma. Die Ptosis bleibt auch während und nach ganz leichtem oberflächlichen Chloräthylrausch bestehen. Für ihre Entstehung konnte keine Erklärung gefunden werden. Vorträge. 
Bachstez: Über den klinisehen, chemischen und histologischen Behind bei der Scintillatio corporis vitrei. (Erschienen in dieser Zeitschrift Bd. 54, Heft 1/2.)

Lindner zeigt an einem Bogenlampenlicht mit Hilfe vorgeschalteter Gläser die Wirkung gekreùzter Zylinder. Daran anschließend bespricht er die Abweichungen des Drehastigmatismus von der richtigen Lage bei unrichtiger Zylinderhöhe. Perzentuell gleiche Stärkenfehler der vorgesetzten Zylinder im Vergleich zum richtigen zeigen bei gleicher Auswärtsdrehung aus der richtigen Lage den gleichen Drehwinkel, d. h.: Dreht man $10^{\circ}$ aus der richtigen Lage und ist der Zylinder im Vergleich zum richtigen

um ein $1 /$ si0 zu niedrig (zu hoch), so beträgt der Richtwinkel immer anstatt $50^{\circ}$

$45,8^{\circ}\left(55,8^{\circ}\right)$, um ein $1 / 10$ zu niedrig (zu hoch), so beträgt der Richtwinkel immer anstatt $50^{*}$

$41,7^{\circ}\left(58,3^{\circ}\right)$, um $1 / 5 \mathrm{zu}$ niedrig (zu hoch), so beträgt der Richtwinkel immer anstatt $50 \mathrm{a}$ $33,9^{\circ}\left(66,1^{\circ}\right)$,

Ophthalmologische Gesellschaft in Wíen.

339

um 1/i zu niedrig (zu hoch), so beträgt der Richtwinkel immer anstatt $50^{\circ}$

$30,5^{\circ}\left(69,5^{\circ}\right)$, um $1 / 2$ zu niedrig (zu hoch), so beträgt der Richtwinkel immer anstatt $50^{\circ}$

$25,7^{\circ}\left(74,3^{\circ}\right)$, um $1 / 2$ zu niedrig (zu hoch), so beträgt der Richtwinkel immer anstatt $50^{\circ}$ $18,9^{\circ}\left(81,9^{\circ}\right)$, um $2 / 3$ zu niedrig (zu hoch), so beträgt der Richtwinkel immer anstatt $50^{\circ}$ $14,7^{\circ}\left(85,3^{\circ}\right)$. Z. B. ist der Drehwinkel bei einem Astigmatismus von 3/4 D, dem man 1/1 vorgesetzt hat, genau so hoch wie bei einem Astigmatismus von $6 \mathrm{D}$, dem man $2 \mathrm{D}$ vorgesetzt hat, nämlich. bei $10^{\circ}$ Fehlstellung $14,7^{\circ}$. Oder würde man statt einer halben D. Zylinder bloß 1/i $\mathrm{D}$ vorgesetzt haben, so ware der Richtwinkel bei $10^{\circ}$ Fehlstellung statt $50^{\circ}$ bloß $18,9^{\circ}$. Diesen selben Winkel bekäme man bei einem Astigmatismus von $4 \mathrm{D}$, dem man $2 \mathrm{D}$ vorgesetzt hat usw. Man ersieht aus diesen wenigen Zifíern sofort, bis zu welcher Zylinderhöhe die Drehprobe noch auf $\mathrm{x} / 4 \mathrm{D}$ bzw. 1/2 D genau ist und daß dieser Probe ein großer Wert mit Ausnahme der letzthin besprochenen Fälle ganz besonders für die Astigma-tismen $\pi$ nter $1 \mathrm{D}$ zukommt.

Der Unterschied im Richtwinkel ist unter sonst gleichen Bedinguogen umso höher, je kleiner der gewählte Drehwinkel ist. Man nimmt daher für gewöhn-lich die Drehprobe auf 5 oder $10^{\circ}$ vor und nur bei ganz schwachen Zylindernauf $20^{\circ}$. (Eigenbericht.)

Aussprache. Kramer (erscheint ausfühiiich in dieser Zeitschrift.)

Diagnose und Therapie.

B. Beütetnieux

Netzhautablösung und perikorneale nicht perîorierende Sklerektomie. Revue Générale dOphthalmologie Bd. 38

1924

S. 90. Verf. wünscht zu demonstrieren daß die Netzhautabhebung nicht so unheilbar ist

wie gemeinhin geglaubt wird.' Er berichtet über drei Fälle von Heilung bei Myopen (einen alten und zwei neue Fälle). Fall 1: Myop von 9 D

der im April 1912 an einem Auge Netzhautablösung erlitt. Wenige Tage nach dem Ereignis wurde eine Sklerektomie aus-geführt. Die Retina legte sich wieder an. Pat. wurde berufsfähig. Im September 1912 traten neue bedrohliche Erscheinungen für Solutio retinae auf. Eine zweite Sklerektomie wurde ausgeführt wieder mit dem Erfolg der Anlegung der Retina. Seitdem also seit zwölf Jahren ist Pat. geheilt geblieben. Fall 2: Myopin von 18-20 D. 
die im April 1923 Netzhautablösung eines Auges am hinteren Pol erlitt. Sie stellte sich sofort dem Verf. vor

der wenige Tage darauf eine Sklerektomie ausführte. Heilung. Gesichtsfeld Sehvermögen Augenhintergrunds- 